\title{
FDTD Investigations into UWB Radar Technique of Breast Tumor Detection and Location
}

\author{
Hua Wang, Marek E. Bialkowski, Fellow, IEEE, Feng Liu and Stuart Crozier, Member, IEEE
}

\begin{abstract}
In this paper, a finite difference time domain (FDTD) method is applied to investigate capabilities of an ultra-wide band (UWB) radar system to detect and locate a breast tumor. The investigations are divided into three parts. The first part concerns an EM field analysis of a phantom formed by a plastic container with liquid and a small highly reflecting target. In the second part, a three-dimensional numerical breast model is used to perform more advanced studies. In the carried out 3D FDTD simulations, a quasi-plane wave is used as an incident wave. Various time snap shots of the electromagnetic field are recorded to learn about the physical phenomenon of reflection and scattering in different layers of the phantoms. The third part of the investigations concerns a two dimensional (cylindrical) image reconstruction, which is performed by means of 2D FDTD. The obtained results should form the ground for working out suitable guidelines for designing an optimal microwave breast imaging apparatus based on the UWB radar technique.
\end{abstract}

Index Terms-Breast Tumor Detection and Location, UWB Radar system, FDTD.

\section{INTRODUCTION}

$\mathrm{R}_{\mathrm{h} w \mathrm{c}}^{\mathrm{c}}$ ecently, various passive and active microwave techniques have been proposed as a viable alternative to X-ray mammography for early detection of breast cancer. These include: passive microwave radiometry [1], [2], hybrid microwave-induced acoustic imaging [3], microwave tomography [4] and ultra-wideband microwave radar technique [5-10]. The passive microwave radiometry relies on temperature differences between malignant and normal breast tissue. The hybrid approach uses ultrasound transducers to detect pressure waves generated by tumor expansion due to microwave heating. In the tomographic approach, a nonlinear inverse scattering problem is solved to obtain spatial distribution of dielectric properties in the breast. The ultra wideband microwave radar uses short duration pulses to detect the presence and location of significant backscatter from malignant tissue.

The basis for the active microwave techniques of breast cancer detection and location is the significant contrast in dielectric properties of normal and malignant breast tissue at this particular frequency spectrum [11-13]. At this frequency range tumours and muscle tissues rich of water exhibit higher dielectric properties than low water contained tissues such as

The authors are with the School of Information Technology and Electrical Engineering, The University of Queensland, St. Lucia, 4072, Queensland, Australia; phone: +61-7-336-54510; fax: +61-7-336-54999; (e-mail: \{hwang, meb, feng, stuart\}@itee.uq.edu.au). fat forming the major part of normal breast tissue. For the normal breast tissues, dielectric properties vary only $\pm 10 \%$ around the relative permittivity $\varepsilon_{r}=9$ and the conductivity $\sigma=$ $0.4 \mathrm{~S} / \mathrm{m}$. For the malignant tumours, the relative permittivity is about 50 and the conductivity is approximately $7 \mathrm{~S} / \mathrm{m}$. These distinctively different dielectric characteristics are the source of backscattered signal, which can be studied using UWB radar techniques to track the position and size of major dielectric contrast interface.

An UWB microwave radar technique for detecting and locating a breast tumour is the subject of investigations in this paper. We emulate the operation of a practical UWB imaging system which is based on a step-frequency synthesized pulse technique [5], [6], [8], [9], [10]. This system consists of a wideband microwave source, a mechanical or electronic antenna scanner, a receiver and a PC controller/processor. In this system the time domain pulse is obtained via stepping frequency over a wide frequency band. A receiver performs measurements of reflection coefficient in frequency domain and its time/space domain equivalent is obtained by applying an Inverse FFT. Using a mechanical scanning subsystem, measurements are repeated for various locations of a UWB probe antenna. In its electronic equivalent, this function is performed by activating electronically various UWB array antenna elements. Next, an image is created by combining all of the processed results coming from different transmitting/receiving antenna locations. In order to enhance the detection process various signal detection enhancement techniques such as confocal microwave imaging [5], space-time beamforming [6] or time-reverse wave focusing [7] can be employed. Because the microwave imaging involves a number of undesired phenomena such as internal reflections in antenna elements, reflections and scattering at the interface between an antenna and an imaged, it is important to carry out full EM analysis of these processes using realistic phantoms. The results of such an analysis can be useful to work out guidelines for designing and developing an optimal UWB radar imaging system for breast cancer detection.

\section{METHODOLOGY}

In the work presented here, we apply a finite difference time domain (FDTD) method [14] to obtain visualization of reflection and scattering in various layers of the breast phantom. The choice of the FDTD method is motivated by the 
fact that it has a distinct advantage of handling field-sample interactions in the time domain. An understanding of these interactions becomes of significant importance, as microwave imaging is moving towards the search of better signal to noise ratio (SNR) and image resolution. In the results presented in this paper, two cases are considered. One is in which, the breast is modeled by a simple phantom in the form of a plastic circular cylindrical container with liquid or air and a highly reflecting target. The second phantom is a breast phantom obtained from detailed dielectric properties of tissues. Each of them is illuminated by a quasi plane wave. In practice, this kind of illumination can be achieved using a large horn antenna or a planar array of in-phase fed wide band antenna elements. Images of field distribution at different time snap shots are collected to the purpose of investigating the wave incidence, reflection and scattering.

\section{A. FDTD method}

The mathematical model of the FDTD method is based on Faraday and Ampere's laws, which provide a system of equations that form an independent set of coupled relationships between time-varying electric and magnetic fields.

$$
\begin{array}{ll}
\nabla \cdot D=\rho & \nabla \cdot B=0 \\
\nabla \times E=-\frac{\partial B}{\partial t} & \nabla \times H=J+\frac{\partial D}{\partial t}
\end{array}
$$

The FDTD method resolves these equations in the time domain by applying central differences to time and space derivatives. With respect to the three dimensional analysis,

$$
\begin{aligned}
& \left.\mathrm{H}_{\mathrm{x}}\right|_{\mathrm{i}, \mathrm{j}+1 / 2 \mathrm{k}+1 / 2} ^{\mathrm{n}+1 / 2}=\left.C_{h 1} \mathrm{H}_{\mathrm{x}}\right|_{\mathrm{i}, \mathrm{j}+1 / 2 \mathrm{k}+1 / 2} ^{\mathrm{n}}+C_{h 2}\left(\frac{\frac{\left.\mathrm{E}_{y}\right|_{\mathrm{i}, \mathrm{j}+1 / 2 \mathrm{k}+1} ^{\mathrm{n}}-\left.\mathrm{E}_{y}\right|_{\mathrm{i}, \mathrm{j}+1 / 2 \mathrm{k}} ^{\mathrm{n}}}{\Delta \mathrm{z}}}{\frac{\left.\mathrm{E}_{\mathrm{z}}\right|_{\mathrm{i}, \mathrm{j}+1, \mathrm{k}+1 / 2} ^{\mathrm{n}} \mathrm{E}_{\mathrm{z}} \mathrm{l}_{\mathrm{i}, \mathrm{j}, \mathrm{k}+1 / 2}^{\mathrm{n}}}{\Delta y}}\right) \\
& \left.\mathrm{H}_{y}\right|_{\mathrm{i}+1 / 2 \mathrm{j}, \mathrm{k}+1 / 2} ^{\mathrm{n}+1 / 2}=\left.C_{h 1} \mathrm{H}_{\mathrm{y}}\right|_{\mathrm{i}+1 / 2 \mathrm{j}, \mathrm{k}+1 / 2} ^{\mathrm{n}}+C_{h 2}\left(\frac{\frac{\left.\mathrm{E}_{z}\right|_{\mathrm{i}+1, \mathrm{j}, \mathrm{k}+1} ^{\mathrm{n}}-\left.\mathrm{E}_{z}\right|_{\mathrm{i}, \mathrm{j}, \mathrm{k}+1 / 2} ^{\mathrm{n}}}{\Delta \mathrm{x}}}{\frac{\left.\mathrm{E}_{\mathrm{x}}\right|_{\mathrm{i}+1 / 2 \mathrm{j}, \mathrm{k}+1} ^{\mathrm{n}}-\left.\mathrm{E}_{\mathrm{x}}\right|_{\mathrm{i}+1 / 2 \mathrm{j}, \mathrm{k}} ^{\mathrm{n}}}{\Delta \mathrm{z}}}\right) \\
& \left.\mathrm{H}_{z}\right|_{\mathrm{i}+1 / 2, \mathrm{j}+1 / 2 \mathrm{k}} ^{\mathrm{n}+1 / 2}=\left.C_{h 1} \mathrm{H}_{z}\right|_{\mathrm{i}+1 / 2, \mathrm{j}+1 / 2 \mathrm{k}} ^{\mathrm{n}}+C_{h 2}\left(\frac{\left.\mathrm{E}_{x}\right|_{\mathrm{i}+1 / 2 \mathrm{j}+1, \mathrm{k}} ^{\mathrm{n}}-\left.\mathrm{E}_{x}\right|_{\mathrm{i}+1 / 2 \mathrm{j}, \mathrm{k}} ^{\mathrm{n}}}{\Delta \mathrm{y}}\right)
\end{aligned}
$$$$
\left.\mathrm{E}_{\mathrm{x}}\right|_{\mathrm{i}+1 / 2, \mathrm{j}, \mathrm{k}} ^{\mathrm{n}+1}=\left.C_{e 1} \mathrm{E}_{\mathrm{x}}\right|_{\mathrm{i}+1 / 2, \mathrm{j}, \mathrm{k}} ^{\mathrm{n}}+\left.\frac{\Delta \mathrm{t}}{\varepsilon} \mathrm{J}_{\mathrm{x}}\right|_{i+1 / 2, j, k} ^{\mathrm{n}+1 / 2}
$$$$
+C_{e 2}\left(\frac{\left.\mathrm{H}_{z}\right|_{\mathrm{i}+1 / 2, \mathrm{j}+1 / 2 \mathrm{k}} ^{\mathrm{n}+1 / 2}-\left.H_{z}\right|_{\mathrm{i}+1 / 2, \mathrm{j}-1 / 2 \mathrm{k}} ^{\mathrm{n}+1 / 2}}{\Delta \mathrm{y}}-\frac{\left.\left.\mathrm{H}_{\mathrm{y}}\right|_{\mathrm{i}+1 / 2, \mathrm{j}, \mathrm{k}+1 / 2} ^{\mathrm{n}+1 / 2}{ }_{\mathrm{y}}\right|_{\mathrm{i}+1 / 2, \mathrm{j}, \mathrm{k}-1 / 2} ^{\mathrm{n}+1 / 2}}{\Delta y}\right)
$$$$
\left.\mathrm{E}_{\mathrm{y}}\right|_{\mathrm{i}, \mathrm{j}+1 / 2 \mathrm{k}} ^{\mathrm{n}+1}=\left.C_{e 1} \mathrm{E}_{\mathrm{y}}\right|_{\mathrm{i}, \mathrm{j}+1 / 2 \mathrm{k}} ^{\mathrm{n}}+\frac{\Delta \mathrm{t}}{\varepsilon} \mathrm{J}_{\mathrm{y}} \mid \begin{aligned}
& \mathrm{n}+1 / 2 \\
& i, j+1 / 2, k
\end{aligned}
$$$$
+C_{e 2}\left(\frac{\left.\left.\mathrm{H}_{x}\right|_{\mathrm{i}, \mathrm{j}+1 / 2 \mathrm{k}+1 / 2} ^{\mathrm{n}+1 / 2} H_{x}\right|_{\mathrm{i}, \mathrm{j}+1 / 2 \mathrm{k}-1 / 2} ^{\mathrm{n}+1 / 2}}{\Delta \mathrm{z}} \frac{\left.\mathrm{H}_{\mathrm{z}}\right|_{\mathrm{i}+1 / 2, \mathrm{j}+1 / 2 \mathrm{k}} ^{\mathrm{n}+1 / 2}-\left.H_{\mathrm{z}}\right|_{\mathrm{i}-1 / 2, \mathrm{j}+1 / 2 \mathrm{k}} ^{\mathrm{n}+1 / 2}}{\Delta \mathrm{x}}\right)
$$

$\left.\mathrm{E}_{\mathrm{z}}\right|_{\mathrm{i}, \mathrm{j}, \mathrm{k}+1 / 2} ^{\mathrm{n}+1}=\left.C_{e 1} \mathrm{E}_{\mathrm{y}}\right|_{\mathrm{i}, \mathrm{j}, \mathrm{k}+1 / 2} ^{\mathrm{n}}+\frac{\Delta \mathrm{t}}{\varepsilon} \mathrm{J}_{\mathrm{y}} \mid \begin{aligned} & \mathrm{n}+1 / 2 \\ & \mid i, j, k+1 / 2\end{aligned}$

$$
\begin{gathered}
+C_{e 2}\left(\frac{\left.\left.\mathrm{H}_{y}\right|_{\mathrm{i}+1 / 2, \mathrm{j}, \mathrm{k}+1 / 2} ^{\mathrm{n}+1 / 2} H_{y}\right|_{\mathrm{i}-1 / 2, \mathrm{j}, \mathrm{k}+1 / 2} ^{\mathrm{n}+1 / 2}}{\Delta \mathrm{x}} \frac{\left.\mathrm{H}_{\mathrm{x}} \mathrm{l}_{\mathrm{i}, \mathrm{j}+1 / 2 \mathrm{k}+1 / 2}^{\mathrm{n}+1 / 2}{ }_{\mathrm{x}}\right|_{\mathrm{i}, \mathrm{j}-1 / 2 \mathrm{k}+1 / 2} ^{\mathrm{n}+1 / 2}}{\Delta \mathrm{y}}\right) \\
\mathrm{C}_{\mathrm{h} 1}=1, \quad \mathrm{C}_{\mathrm{h} 2}=\frac{\Delta \mathrm{t}}{\mu}, \quad \mathrm{C}_{\mathrm{e} 1}=\frac{2 \varepsilon-\sigma \Delta t}{2 \varepsilon+\sigma \Delta t}, \quad C_{e 2}=\frac{2 \Delta t}{(2 \varepsilon+\sigma \Delta t)}
\end{gathered}
$$

these equations are given by (2):

In the discrete FDTD computational domain in Cartesian coordinates, the dimensions of the Yee cell are defined by $\Delta x$, $\Delta y$ and $\Delta z$, as small as $0.5 \mathrm{~mm}$ to reduce the "staircase" error [15] on the curved surface of plastic container and copper. The relative permeability $\mu_{r}(x, y, z)$, relative permittivity $\varepsilon_{r}(x, y, z)$ and material conductivity $\sigma(x, y, z)$ are defined at the center of each cell are stored in a look-up table for the purpose of efficient memory utilization.

For the two-dimensional (cylindrical) case, with TE excitation with respect to the direction of propagation, the equations are given by (3).

$$
\begin{aligned}
& \left.\mathrm{H}_{\mathrm{x}}\right|_{\mathrm{i}, \mathrm{j}+1 / 2 \mathrm{k}+1 / 2} ^{\mathrm{n}+1 / 2}=\left.C_{h 1} \mathrm{H}_{\mathrm{x}}\right|_{\mathrm{i}, \mathrm{j}+1 / 2 \mathrm{k}+1 / 2} ^{\mathrm{n}}-C_{h 2}\left(\frac{\left.\mathrm{E}_{\mathrm{z}}\right|_{\mathrm{i}, \mathrm{j}+1, \mathrm{k}+1 / 2} ^{\mathrm{n}}-\left.\mathrm{E}_{\mathrm{z}}\right|_{\mathrm{i}, \mathrm{j}, \mathrm{k}+1 / 2} ^{\mathrm{n}}}{\Delta y}\right) \\
& \left.\mathrm{H}_{\mathrm{y}}\right|_{\mathrm{i}+1 / 2, \mathrm{j}, \mathrm{k}+1 / 2} ^{\mathrm{n}+1 / 2}=\left.C_{h 1} \mathrm{H}_{\mathrm{y}}\right|_{\mathrm{i}+1 / 2, \mathrm{j}, \mathrm{k}+1 / 2} ^{\mathrm{n}}+C_{h 2}\left(\frac{\mathrm{E}_{z} \mathrm{i}_{\mathrm{i}+1, \mathrm{j}, \mathrm{k}+1}^{\mathrm{n}}-\mathrm{E}_{z} \mathrm{n}_{\mathrm{i}, \mathrm{j}, \mathrm{k}+1 / 2}^{\mathrm{n}}}{\Delta \mathrm{x}}\right) \\
& \left.\mathrm{E}_{\mathrm{z}}\right|_{\mathrm{i}, \mathrm{j}, \mathrm{k}+1 / 2} ^{\mathrm{n}+1}=\left.C_{e 1} \mathrm{E}_{\mathrm{y}}\right|_{\mathrm{i}, \mathrm{j}, \mathrm{k}+1 / 2} ^{\mathrm{n}}+\frac{\Delta \mathrm{t}}{\varepsilon} \mathrm{J}_{\mathrm{y}} \mid \begin{array}{l}
\mathrm{n}+1 / 2 \\
i, j, k+1 / 2
\end{array} \\
& +C_{e 2}\left(\frac{\left.\mathrm{H}_{y}\right|_{\mathrm{i}+1 / 2 \mathrm{j}, \mathrm{k}+1 / 2} ^{\mathrm{n}+1 / 2}-\left.H_{y}\right|_{\mathrm{i}-1 / 2 \mathrm{j}, \mathrm{k}+1 / 2} ^{\mathrm{n}+1 / 2}}{\Delta \mathrm{x}}-\frac{\left.\mathrm{H}_{\mathrm{x}}\right|_{\mathrm{i}, \mathrm{j}+1 / 2 \mathrm{k}+1 / 2} ^{\mathrm{n}+1 / 2}-\left.H_{\mathrm{x}}\right|_{\mathrm{i}, \mathrm{j}-1 / 2 \mathrm{k}+1 / 2} ^{\mathrm{n}+1 / 2}}{\Delta \mathrm{y}}\right)
\end{aligned}
$$

Finally, Berenger's PML (Perfectly Matched Layer) [16] is employed as an absorbing boundary condition. In addition, the Message Passing Interface (MPI) library [17] is used so that a parallel computing frame is implemented. As a result of these steps, the simulation process for each of the two investigated cases takes less than 20 mins on a cluster of three servers each with 2 XEON $3.6 \mathrm{GHz}$ processors. The results are generated and visualized by recording the electric fields at various positions in real time. Image reconstruction from the backscattered signal presents contrastive energy distribution due to different target. By using this approach, the details of wave propagation, reflection and scattering can be analysed. In particular, this EM field analysis can allow for investigating the subtle features of the detection of very small targets.

\section{B. System model}

The FDTD model aims at emulating a microwave imaging system [18] that employs a synthesized pulse technique. The FDTD model for the first analysed phantom that includes a plastic container and a small highly reflecting target is shown in Fig.1. 


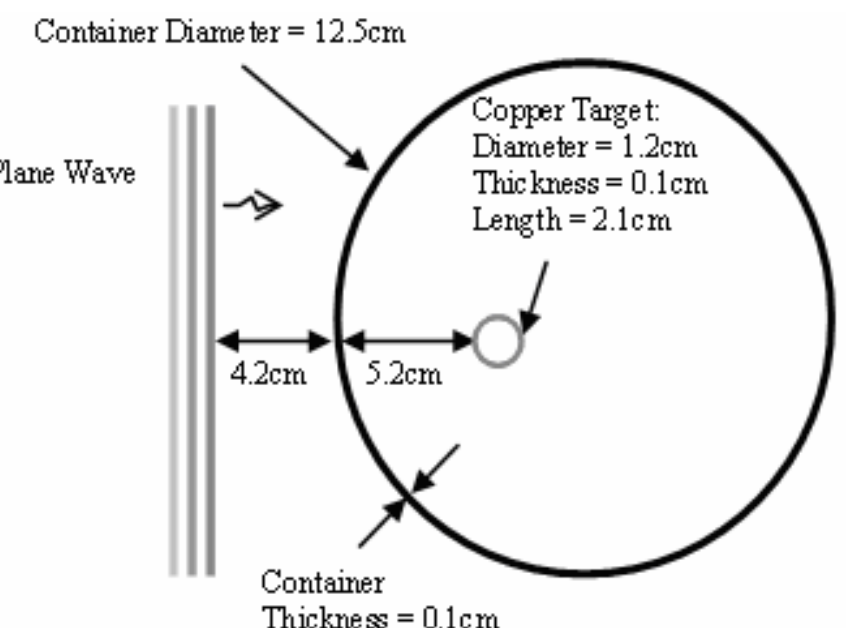

Fig. 1. The configuration of a plastic container with liquid and a highly reflecting target, as used in an experimental setup described in [18].

In the experimental system, reflection coefficient measurements are performed in the frequency domain over an assumed wide frequency band for many locations of a probe antenna over a planar surface. For each location of the probe antenna, the measured data is transformed to the time domain by using an Inverse FFT. In the FDTD model a quasi planar wave is launched having constant power spectral density over the assumed wide frequency band.

Fig.2. shows a relationship between the frequency domain and time domain representations of the signals incident on the imaged object.

(a)

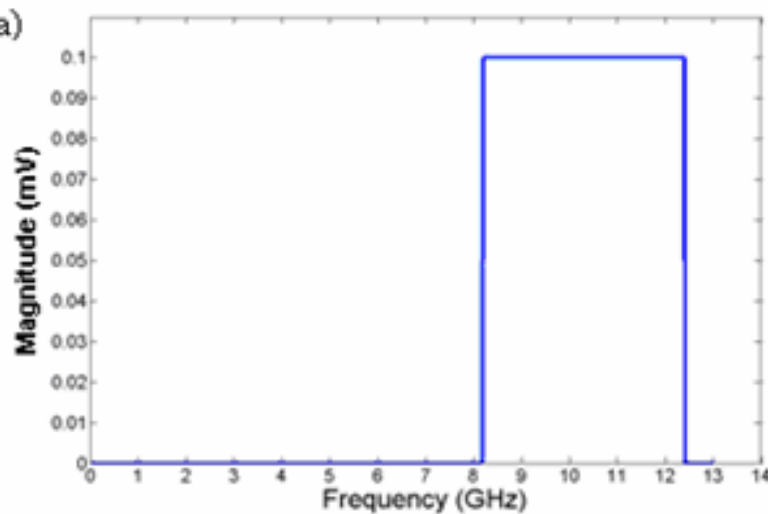

(b)

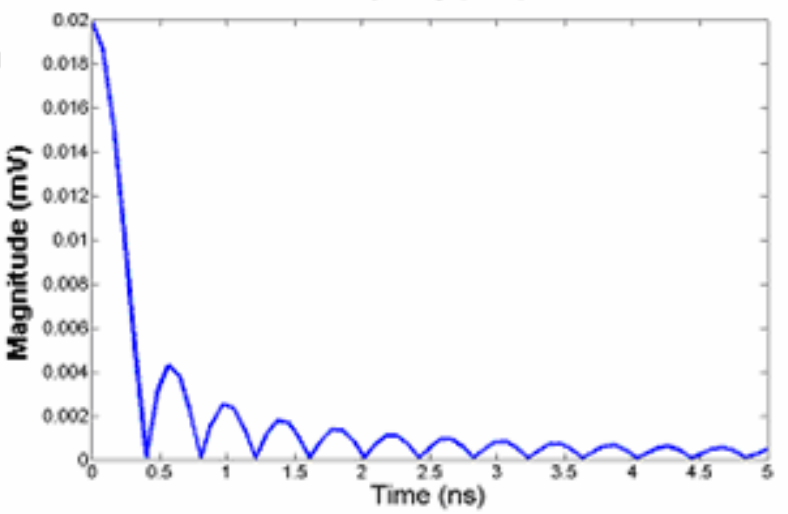

Fig. 2. Synthesized pulse, (a) frequency domain (b) time domain representation.
Note that in the current experimental system as described in [18] the frequency band is assumed to be between 8.4 to 12.4 $\mathrm{GHz}$. Both FDTD simulations and the experimental setup are easily extendable to a larger frequency band to obtain a higher resolution of target location.

In the first experimental setup, shown in Fig.1, the imaged object consists of a circular cylindrical plastic container $\left(\varepsilon_{r}=\right.$ $2.5)$ with a diameter of $12.5 \mathrm{~cm}$ with thickness of $1 \mathrm{~mm}$. The container is assumed to be filled with oil $\left(\varepsilon_{r}=4.0\right)$. A solid material representing a target tumour is located inside the container. A highly conductive object $(\sigma=1 \mathrm{e} 6)$, in the form of a copper pipe of diameter $1.2 \mathrm{~cm}$ and length of $2.1 \mathrm{~cm}$ is located close to the centre of the plastic container.

In the second experiment, a detailed breast phantom obtained with different dielectric properties of normal and malignant breast tissue is investigated. This phantom contains the outside skin to observe the dominant reflection from the skin, while the internal area is filled with breast fat, and a spherical tumour tissue with $6 \mathrm{~mm}$ diameter located inside. The dielectric properties are $\varepsilon_{r}=6.89, \sigma=32.25$ for skin, $\varepsilon_{r}=$ 0.67, $\sigma=4.0$ for breast fat and $\varepsilon_{r}=9.19, \sigma=44.12$ for malignant tissue, which is equivalent to muscle as presented above. A pulse, as shown in Fig.2b, is launched towards the phantom.

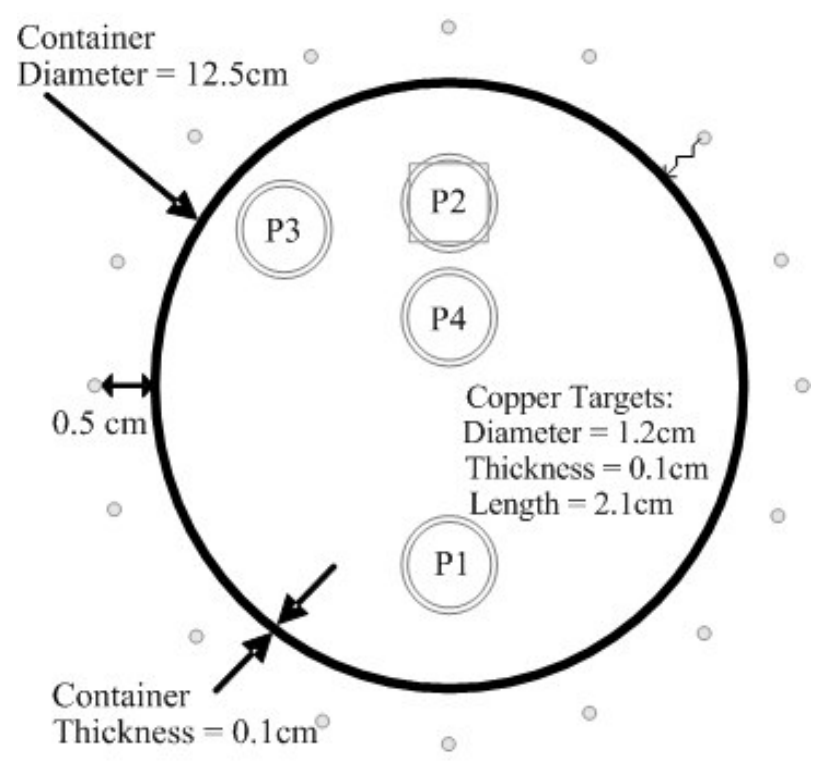

Fig. 3. The configuration of a plastic container with highly reflecting targets of different positions, sizes and shapes.

The $3^{\text {rd }}$ case concerns the $2 \mathrm{D}$ cylindrical configurations when a TE wave is incident. In this model, as shown in Fig. 3, instead of simulating an antenna array as previously defined, transmitters/receivers are distributed around the breast phantom. Targets of different positions (P1, P2, P3 and P4), sizes and shapes are assumed. The scanning process is executed by activating individual antennas. In this case, one antenna launches a pulse and receives a backscattered signal synchronously. This process is repeated for the remaining (15) antennas. After obtaining the measurement for 16 antennas, each intensity record along time domain is projected onto 
circular coordinate system to reconstruct the dielectric property distribution of the target.

\section{RESULTS AND DISCUSSION}

The obtained FDTD simulation results allow for animation of the entire process of wave launching, propagation, reflection and scattering. Here, we present only selected time snap shots of the field distribution representing this entire process. Fig. 4 illustrates the incidence, reflection and scattering phenomenon at different layers of the first phantom. Fig. 4 (a) shows the wave propagation in free space prior to the incidence on the plastic container. A major reflection at the plastic container interface is observed in Fig. 4b. Fig. 4c reveals the field distribution before the wave strikes the copper target. In the next slides, the wave reflection and scattering process from the target takes place. Before the reflected and scattered waves reach the outside region of the
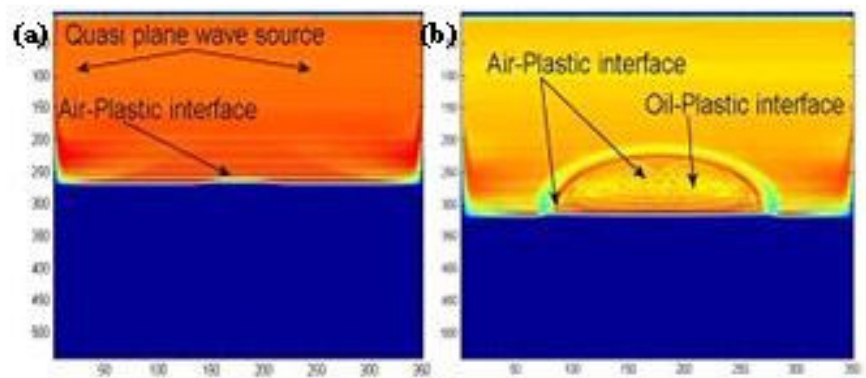

(c)
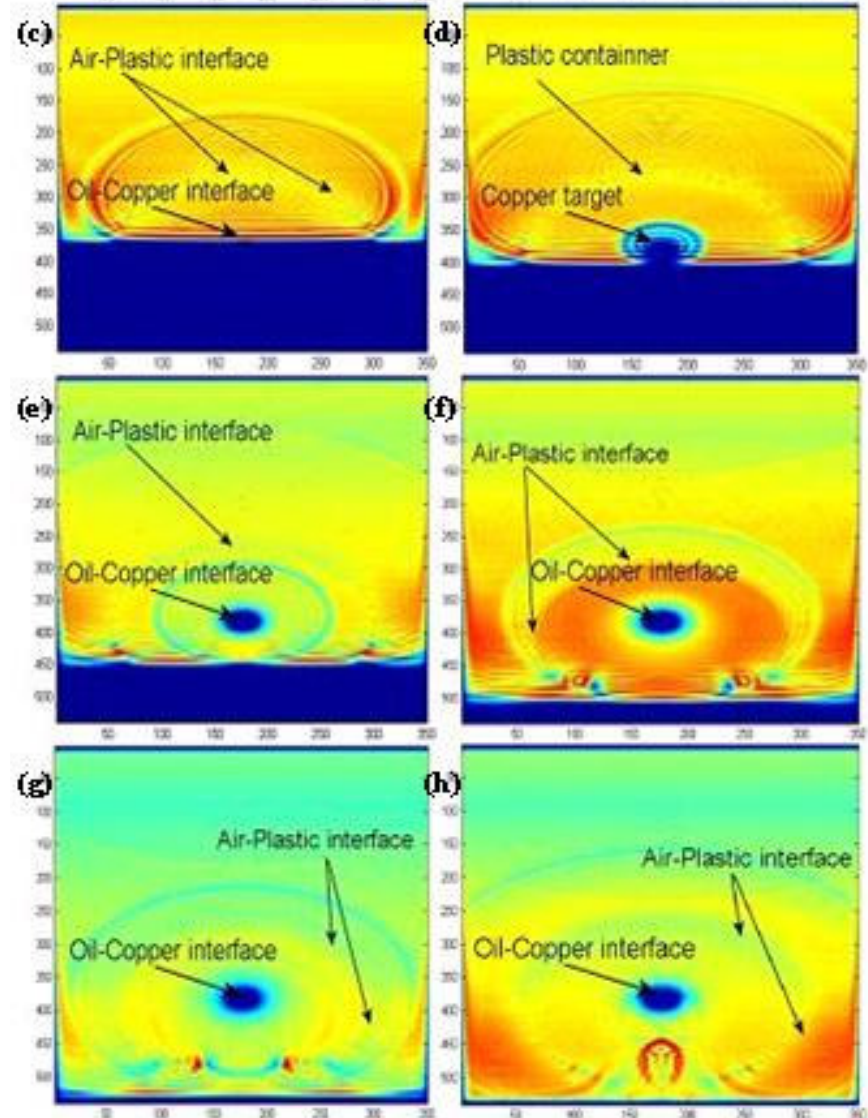

Fig. 4. Time snap shots showing the process of wave propagation in the firs phantom.

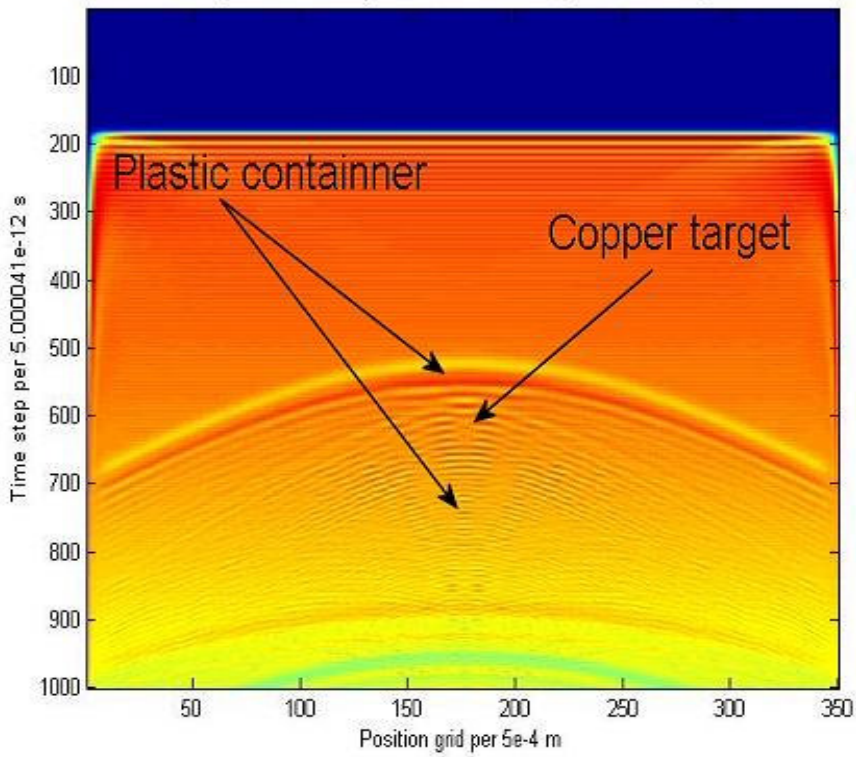

Fig. 5. Electric field distribution when backscattered wave reaches the position at which an incident wave was launched.

phantom, an additional reflection and scattering phenomenon at the inner interface between the liquid and the plastic container takes place. This additional scattering obscures the capability of target detection outside the phantom.

Figure 5 presents the field distribution at the time the reflected waves reach the position, from which the signal was launched. The obtained image shows that the back scattered wave (from the target) is obscured by other waves caused by other reflections and scattering in the phantom. Much a clearer picture of the backscattered wave is shown in Figure 6. It was obtained by subtracting the electric field distribution obtained without the target inside the phantom.

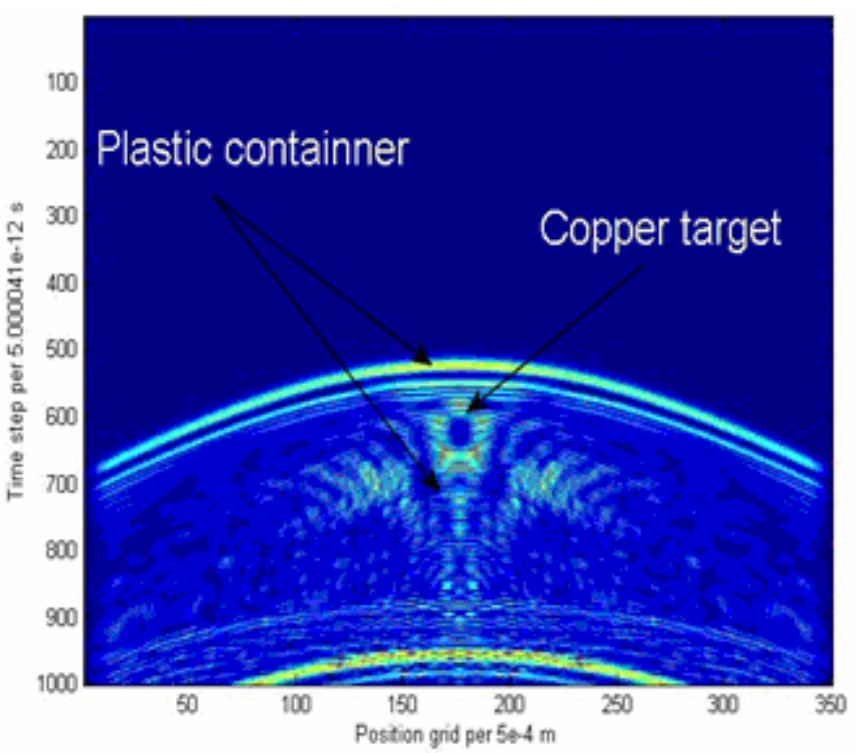

Fig. 6. Received backscatter obtained from subtracting the electric field distribution without the target.

The results presented in Figure 7 concern the second phantom, which assumes more realistic shape, size and dielectric properties of the breast. The results presented in 

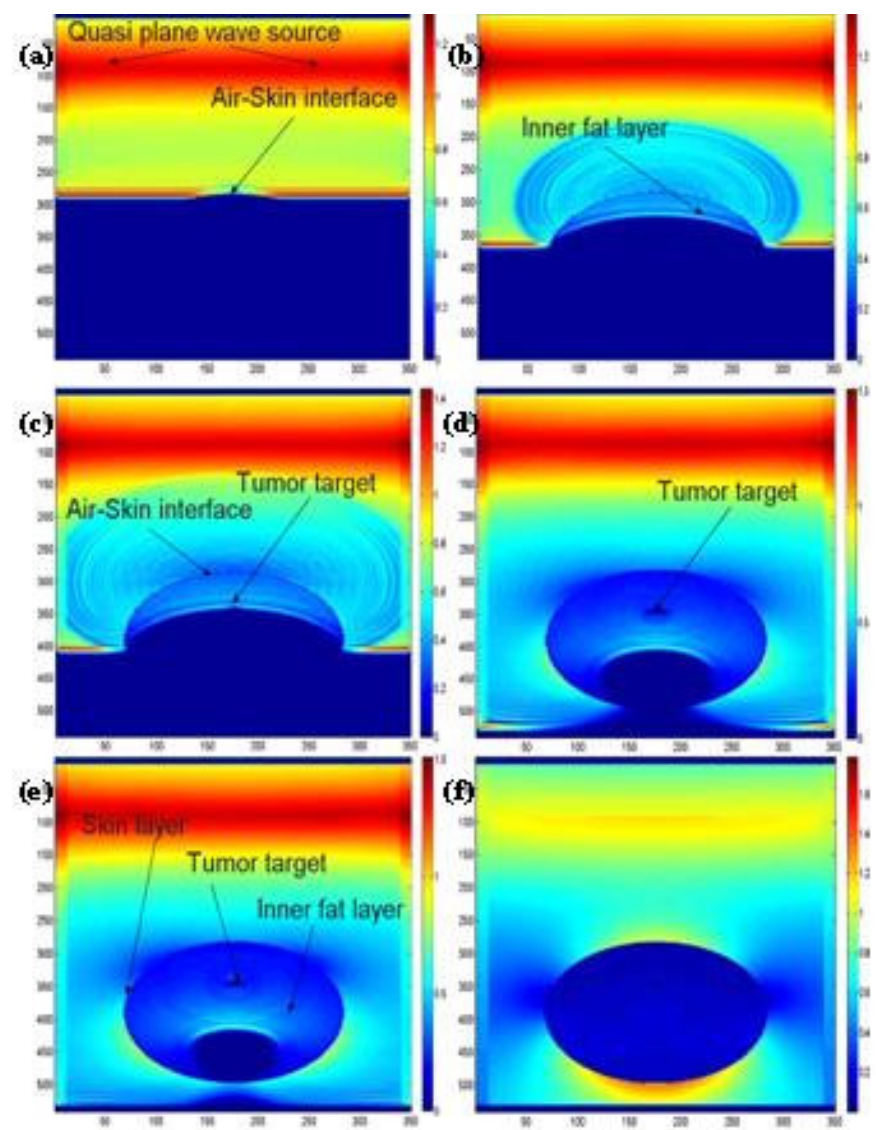

Fig. 7. Wave propagation within the 3D breast phantom

Figure 7 reveal that the reflection from the air-skin interface is dominant. The wave reflected and scattered from the small tumor tissue is obscured by the random scattering caused by the skin interface and the non uniform composition of the breast tissues. Compared with the first phantom with plastic container and a copper target, the tumor target in the second phantom is less visible. Many methods have been developed
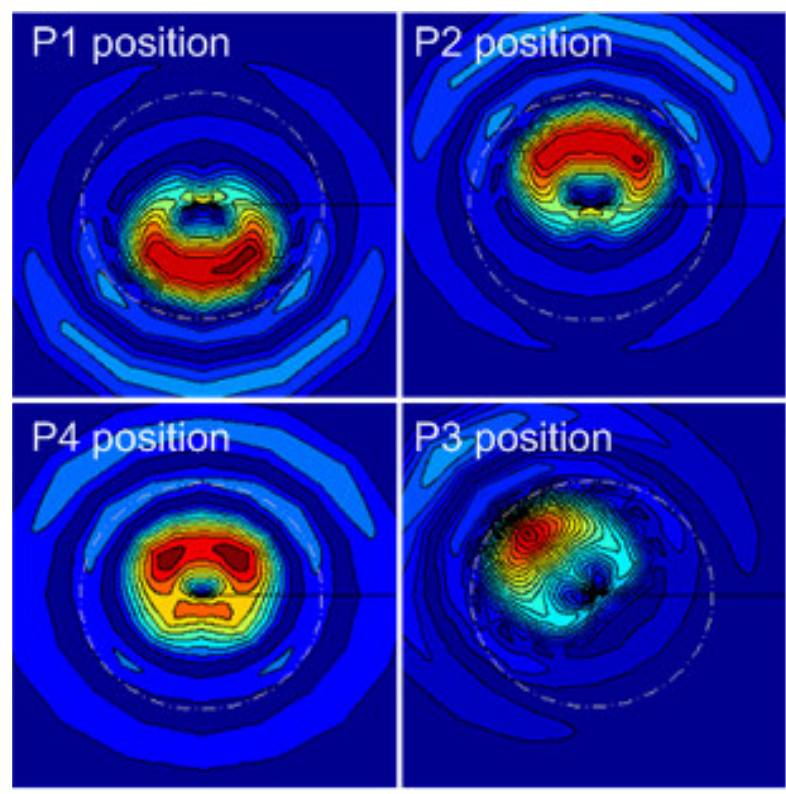

Fig. 8. Reconstructed images for circle targets at different positions (P1, P2, P3 and P4). to enhance the target scattering effects by using dielectric liquids around the breast to reduce strong reflection from the surface of skin layer.

The remaining Figures 8-10 concern the 2D phantom and demonstrate the capability of high contrast interface visualization of dielectric properties. Figure 8 presents different maps of field distribution according to 4 positions (P1, P2, P3 and P4) of targets. The obtained locations of high scatter match the actual location of the targets. As seen in Figure 8, with respect to the penetrating capabilities of EM waves in dielectric, lower targets, such as at P3 position, provide better resolution. Because of iterative reflections and scattering inside the phantom, inner targets are obscured. This problem can be overcome by wave focusing and target calibration. The field distribution obtained herein for different positions of the targets can be helpful to develop more accurate image reconstruction methods.
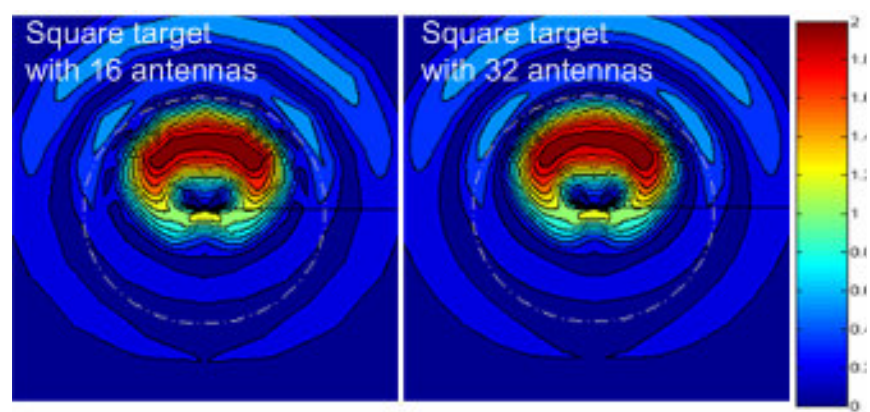

Fig. 9. Reconstructed images for square targets obtained using 16 and 32 antennas.

In Figure 9, the right image provides more detailed information and it is obtained using 32 instead of 16 antenna elements on the left image.

Figure 10 shows the results when the targets feature different size. As observed in Figure 10, larger size targets have a larger effect on the propagation of the EM wave. More energy concentrates around the contrast interface.
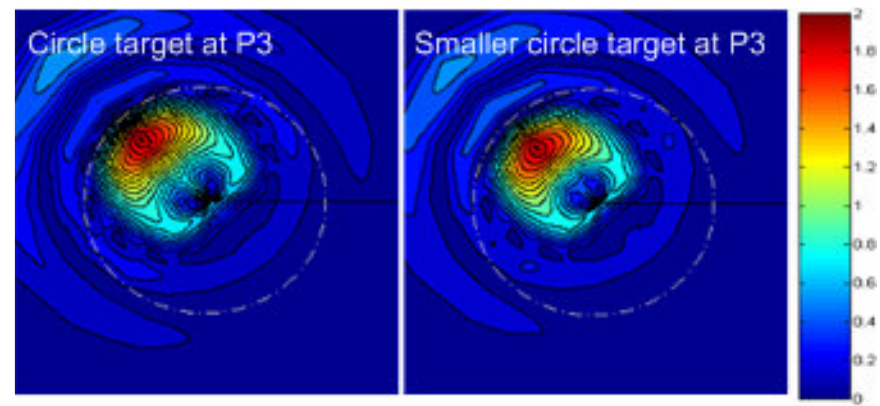

Fig. 10. Reconstructed images for circular cylindrical targets with smaller size using 16 antennas.

The reconstructed images of different targets obey general principles of inverse problem. The more information provided, the more accurate reconstructed image becomes. For the specific problems considered herein, more antennas and a larger frequency bandwidth, as used in UWB radar technique, can provide better resolution of the dielectric targets. 


\section{CONCLUSION}

In this paper, various phantoms have been analyzed with the FDTD algorithm to investigate the capability of an ultra-wide band microwave radar system for detection and location of breast tumor. The first phantom is a circular cylindrical plastic container with liquid and a small highly reflecting target. The other one is a three-dimensional numerical breast model obtained with practical dielectric properties of tissues in the breast. In order to accelerate computations an FDTD algorithm for use on a cluster of parallel processors has been developed. Numerical calculations have been performed to investigate the phenomenon of wave reflection and scattering in various layers of the two phantoms. In the carried out computer simulations, an incident wave in the form of a quasi planar wave has been assumed. The third considered problem has concerned the use of 2D FDTD to investigate image reconstruction capabilities of UWB radar. It has been shown that better quality target resolution can be achieved using more antenna elements (or positions in a mechanical scanning system) around the circular cylindrical phantom.

In terms of the studies of the reflection and scattering phenomenon in these models and the investigation of target detecting capability so far, future work will concern other problems associated with breast tumor location and detection and will include amongst the other such issues as inclusion of other types of waves having capability of focussing on the target to achieve a better quality detection of the malignant tissues.

\section{REFERENCES}

[1] K.L Carr, "Microwave radiometry: Its importance to the detection of cancer", IEEE Trans. Microwave Theory and Tech., vol. 37, no. 12, pp. 1862-1869, Dec. 1989.

[2] B. Bocquet et al., "Microwave radiometric imaging at $3 \mathrm{GHz}$ for the exploration of breast tumors", IEEE Trans. Microwave Theory and Tech., vol. 38, no. 6, pp. 7910793, June 1990.

[3] L.V. Wang et al.,"Microwave-induced acoustic imaging for biological tissues", Rev. Sci. Instrum., vol. 70, pp. 3744-3748, 1999.

[4] A. E. Bulyshev, S. Y. Semenov, A. E. Souvorov, R. H. Svenson, A. G. Nazarov, Y. E. Sizov, and G. P. Tatsis, "Computational modelling of three-dimensional microwave tomography of breast cancer," IEEE Trans. Biomed. Eng., vol. 48, no. 9, pp. 1053-1056, Sep. 2001.

[5] E. C. Fear, X. Li, S. C. Hagness, and M. A. Stuchly, "Confocal microwave imaging for breast tumor detection: Localization in three dimensions," IEEE. Trans. Biomed. Eng., vol. 49, no. 8, pp. 812-822, Aug. 2002.

[6] E. J. Bond, X. Li, S. C. Hagness, and B. D. Van Veen, "Microwave imaging via space-time beamforming for early detection of breast cancer," IEEE Trans. Antennas Propag., vol. 51, no. 8, pp. 1690-1705, Aug. 2003.

[7] P. Kosmas and C. M. Rappaport, "Time reversal with the FDTD method for microwave breast cancer detection," IEEE Trans. Microwave Theory and Tech., vol. 53, no. 7, pp. 2317-2323, July 2005.

[8] E. C. Fear, P. M. Meaney, and M. A. Stuchly, "Microwaves for breast cancer detection?," IEEE Potentials, vol. 22, pp. 12-18, 2003.

[9] M. Converse, E. J. Bond, S. C. Hagness, and B. D. Van Veen, "Ultrawideband microwave space-time beamforming for hyperthermia treatment of breast cancer: A computational feasibility study," IEEE
Trans. Microw. Theory Tech., vol. 52, no. 8, pp. 1876-1889, Aug. 2004.

[10] X. Li, S. K. Davis, S. C. Hagness, D. W. van der Weide, and B. D. Van Veen, "Microwave imaging via space-time beamforming: Experimental investigation of tumor detection in multilayer breast phantoms," IEEE Trans. Microwave Theory Tech., vol. 52, pp. 1856-1865, Aug. 2004.

[11] S. Gabriel, R. W. Lau, and C. Gabriel, "The dielectric properties of biological tissues: 11 . Measurements on the frequency range $10 \mathrm{~Hz}$ to 20 GHz," Phys. Med. Biol., vol. 41, pp. 2251-2269, Nov. 1996.

[12] C. Gabriel, S. Gabriel, and E. Corthout, "The dielectric properties of biological tissues: I. Literature survey," Phys. Med. Biol., vol. 41, pp. 2231-2249, Nov. 1996.

[13] W. T. Joines, Y. 2. Dhenxing, and R. L. Jirtle, "The measured electrical properties of normal and malignant human tissues from 50 to 900 MHz," Med. Phys., vol. 21, pp. 547-550, Apr. 1994.

[14] K. S. Yee, "Numerical solution of initial boundary value problems involving Maxwell's equations in isotropic media," IEEE Trans. Antennas Propagation., Vol. AP-14, pp. 302-307, 1966.

[15] A. C. Cangellaris and D. B. Wright, "Analysis of the numerical error caused by the stair-stepped approximation of a conducting boundary in FDTD simulations of electromagnetic phenomena," IEEE Trans. Antennas Propagation., Vol. 39, pp. 1518-1525, Oct. 1991.

[16] J. P. Berenger, "A Perfectly matched layer for the absorption of electromagnetic waves," Journal of Computational Physics., Vol. 114, pp. 185-200, 1994.

[17] C. Guiffaut and K. Mahdjoubi, "A parallel FDTD algorithm using the MPI library," IEEE Antennas and Propagation Magazine, Vol. 43, No. 2, 2001.

[18] M.E. Bialkowski, W.C. Khor, W.C., and S. Crozier, " A planar microwave imaging system with step-frequency synthesized pulse using different calibration methods", Microwave and Optical Technology Letters, 2006, 48, (3), pp. 511-516.

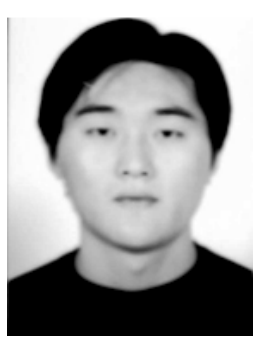

Hua Wang received the B.E. degree of Computer Science and Technology in Nanjing University of Aeronautics and Astronautics, China in 2003.

$\mathrm{He}$ is currently a $\mathrm{PhD}$ candidate in the School of Information Technology and Electrical Engineering, University of Queensland, Australia. His research interests include numerical modeling of electromagnetic problems, parallel computing, and AI optimization algorithms.

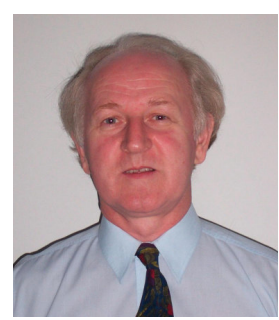

Marek E. Bialkowski (SM'88-F'03) was born in Sochaczew, Poland. He received the M.Eng.Sc. degree (1974) in applied mathematics and the Ph.D degree (1979) in electrical engineering both from the Warsaw University of Technology and a higher doctorate (D.Sc. Eng.) in computer science and electrical engineering from the University of Queensland (2000)

He held teaching and research appointments at universities in Poland, Ireland, Australia, UK, Canada, Singapore, Hong Kong and Switzerland. At present he is a Professor in the School of Information Technology and Electrical Engineering at the University of Queensland. His research interests include antennas for mobile cellular and satellite communications, signal processing techniques for smart antennas, low profile antennas for reception of satellite broadcast TV programs, near-field/far-field antenna measurements, electromagnetic modelling of waveguide feeds and transitions, conventional and spatial power combining techniques, six-port vector network analysers, and medical 
and industrial applications of microwaves. He has published over 400 technical papers, several book chapters and one book.

Prof. Bialkowski was a Corecipient of a 2000 Harold A. Wheeler Applications Prize Honorable Mention for a paper published in the IEEE TRANSACTIONS ON ANTENNAS AND PROPAGATION in 1999. In 2002, he was elected an IEEE Fellow for contributions to the modeling, design, and testing of microwave guiding and radiating structures.

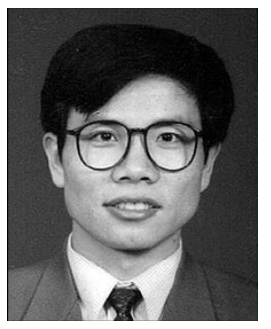

Feng Liu received the Ph.D. degree in biomedical engineering from Zhejiang University in 2000. He is currently working in The School of Information Technology and Electrical Engineering, University of Queensland, as a Senior Research Fellow. He has published about 80 journal articles and conference papers. His research interests include magnetic resonance engineering, bioelectromagnetism and biomechanics.

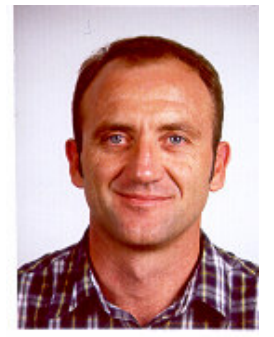

Stuart Crozier (M'93) received the Ph.D. and D.Eng degrees from the University of Queensland, Australia in 1991 and 2002 respectively. He currently holds the position of Professor of Biomedical Engineering in The School of Information Technology and Electrical Engineering. He has published over 110 journal articles and has numerous patents. His research interests include Magnetic Resonance Engineering, bioelectromagnetics and physiological signal measurement and analysis. 